\title{
Truncating Mutation in FOXC2 Gene in Familial Hemorrhoids and Varicose Veins
}

\author{
Janti Qar ${ }^{1}$, Mazhar Salim Al Zoubi ${ }^{2 *}$, Ibrahim M.A. Baydoun ${ }^{1}$, Alaa A. A. Aljabali ${ }^{3}$, Bahaa Al-Trad ${ }^{1}$, \\ Firas Rabi ${ }^{4}$, Khalid M. Al Batayneh ${ }^{1 *}$ \\ ${ }^{1}$ Department of Biological Sciences, Faculty of Science, Yarmouk University, Irbid-21163, Jordan \\ ${ }^{2}$ Department of Basic Medical Sciences, Faculty of Medicine, Yarmouk University, Irbid-21163, Jordan \\ ${ }^{3}$ Faculty of Pharmacy, Yarmouk University, Irbid-21163, Jordan \\ ${ }^{4}$ Department of Clinical Sciences, Faculty of Medicine, Yarmouk University, Irbid-21163, Jordan \\ *Corresponding authors: Khalid Al-Batayenh and Mazhar Al Zoubi \\ albatynehk@yu.edu.jo, mszoubi@yu.edu.jo
}

\begin{abstract}
Hemorrhoids and varicose veins are conditions resulting from loss of vascular integrity and, despite being worldwide health concerns, their pathogenesis has not been clearly defined. Many risk factors have been linked to the development of these complications including diet, defecating habits, alcohol consumption and other physiological factors. There are limited studies involving the possible role of genetic mutations in the development of hemorrhoids and varicose veins. FoxC2 is an important transcription factor that plays many roles in a variety of embryonic developmental processes, including angiogenesis. In the current study, we aimed to investigate the role of the FOXC2 gene variations in the development of familial hemorrhoids and varicose veins in the Jordanian population. Thirty-two samples were collected from eight families manifested hemorrhoids and/or varicose veins conditions. DNA sequencing was performed to screen variation in the FOXC2 gene. Two individuals with severe and early onset of hemorrhoids and varicose veins from the same family showed a frameshift mutation (881'inT) in the coding exon of the FOXC2 gene resulting in a premature stop codon at position $+1386(294$ residues truncated peptide). In conclusion, our results support a possible role of genetic predisposition in the development of hemorrhoids and varicose veins with a frequency of $6 \%$ in the selected population.
\end{abstract}

Keywords-FOXC2, Hemorrhoids, Varicose veins.

\section{INTRODUCTION}

$\mathrm{H}$ emorrhoids refer to the complex of submucosal, fibrovascular, and arteriovenous sinusoids within the normal anorectum, which affects up to $50 \%$ of the population [1,2]. The etiology of hemorrhoids is uncertain, however, potential risk factors that have been suggested include a low-fiber diet, spices, body mass index, defecation time and habits and alcohol consumption [2-7]. In addition, instability of collagen structure and high activity of metalloproteases have been suggested to be associated with the development of hemorrhoids [8-11]. Similarly, limited information is available about the pathophysiology, proper treatment of the hemorrhoids. Therefore, molecular and genetic studies can be helpful to pinpoint the pathogenesis of hemorrhoids [2].

Varicose veins have been defined as twisted and dilated veins with an abnormal diameter. Many factors have been suggested to be associated with the development of varicose veins including older age, gender, family history, pregnancy, obesity and standing or sitting for a long period [12]. In addition, genetic predisposition was found to be associated with the pathogenesis of various chronic venous diseases. For instance, many genetic alterations were associated with venous thrombosis disease [13]. Previous studies supported the genetic predisposition and familial susceptibility to varicose veins [14-18]. For instance, a missense mutation of the COL3A1 gene has been identified in an Ehlers-Danlos syndrome case with varicose veins [19]. Moreover, an increased incidence of chromosomal aberrations has been identified in families with early-onset varicose veins [20]. Interestingly, the D16S520 marker of the FOXC2 gene has been proposed to be associated with the development of varicose veins [21]. Additionally, FOXC2 mutations were linked to primary valve failure in veins of the lower limb with varicose veins [22].

The FOXC2 gene (HGNC:3801) encodes for the Forkhead box C2 transcription factor (FoxC2), also known as forkheadrelated protein (FKHL14) or mesenchyme forkhead 1 protein (MFH1). FoxC2 transcription factor has been identified as a new member of the forkhead family proteins in 1993 by Naoyuki Miur et al and suggested to be involved in the development of embryonic mesenchymal tissues [23]. Later, it has been found that FoxC2 protein (MFH-1) has a crucial role in the aortic arch formation and skeletogenesis, mesodermal neural tube formation, vertebral column and ocular development [24-27]. In addition, FoxC2 inhibits the differentiation of white adipocytes and stimulates that of osteoblasts [28, 29]. Importantly, FoxC2 protein was described as an important factor for the vascular pathogenesis [30]. The role of FoxC2 factor in the angiogenesis process has been 
demonstrated through the induction of the transcription of integrin $\beta 3$ and CXCR4, the key players of the endothelial cell migration [31-34]. Moreover, FoxC2 was described as a modulator in the lymphangiogenesis [35-37]. Interestingly, FoxC2 has shown an important role in tumor development and metastasis through the upregulation of the angiogenesis process [38-40]. Collectively, FoxC2 protein was described as a central regulator and coordinator of the angiogenesis signaling pathway via endothelial cell migration, vascular development and restoration [41]. In a previous study, we sequenced a fragment of the FOXC2 gene spanning from -413 bp position of the 5 '-UTR region to position +655 bp of the coded area [18].

In the current study, we aimed to elucidate the role of the FOXC2 gene variations in the development of hemorrhoids and varicose veins conditions through screening of the FOXC2 exon and 3'-UTR region.

\section{MATERIALS AND METHODS}

\section{A. Subjects and sample collection:}

Thirty-two blood samples were collected from eight families manifesting symptoms of varicose veins and hemorrhoids. In addition, thirty blood samples were collected from a healthy control group. Written consent was obtained from each participant. Relevant medical history was collected and analyzed through a questionnaire. Individuals with infectious history and suspected environmental factors were excluded from this study.

\section{B. Genomic DNA extraction and PCR}

Genomic DNA was extracted from blood samples using Wizard Genomic DNA Extraction Kit (Bio Basic, Canada) according to the manufacturer's instructions. Extracted DNA was stored at $-80^{\circ} \mathrm{C}$ until use. The amplification reaction was carried out using Xp Thermal Cycler (Bioer Tech, China). Three pairs of primers were used to amplify the target sequence $(+444$ to +1784$)$ of the FOXC2 gene (Fig. 1) (Table 1). The amplification conditions were set as the following: Initial denaturation at $95^{\circ} \mathrm{C}$ for ten minutes, 35 cycles of denaturation at $95^{\circ} \mathrm{C}$ for one minute, annealing at the specific $\mathrm{Tm}$ for one minute, extension at $72^{\circ} \mathrm{C}$ for one minute, and followed by final extension $72^{\circ} \mathrm{C}$ at for ten minutes. The amplification reaction was carried out using a $2 \mathrm{X}$ PCR master mix from (New England laboratories, USA).

Table 1. Sets of primers indicating the expected amplicon sizes and annealing temperature $(\mathrm{Tm})$

\begin{tabular}{|c|c|c|c|}
\hline$\underset{\#}{\operatorname{Amp}}$ & Primer sequence & $\begin{array}{l}\text { Tm } \\
\left({ }^{\circ} \mathbf{C}\right)\end{array}$ & size \\
\hline $\begin{array}{c}\text { Amp } \\
1\end{array}$ & $\begin{array}{l}\text { F-5' GACCCGGACTCCTACAACAT 3', } \\
\text { R-5' GAGCGATGAGCCTGTACACC 3, }\end{array}$ & 56 & $\begin{array}{c}576 \\
\text { bp }\end{array}$ \\
\hline$\underset{2}{\mathrm{Amp}}$ & $\begin{array}{l}\text { F-5' GAGCGATGAGCCTGTACAC 3, } \\
\text { R-5' GAAATACTGACGTGTCCCG 3, }\end{array}$ & 55 & $\begin{array}{c}515 \\
b p\end{array}$ \\
\hline$\underset{3}{\operatorname{Amp}}$ & $\begin{array}{l}\text { F-5' CAACGTGCGGGAGATGTTCAAC3, } \\
\text { R-5' CTACCAGGACGGCTGTGCTGTG 3', }\end{array}$ & 60 & $\begin{array}{c}463 \\
b p\end{array}$ \\
\hline
\end{tabular}

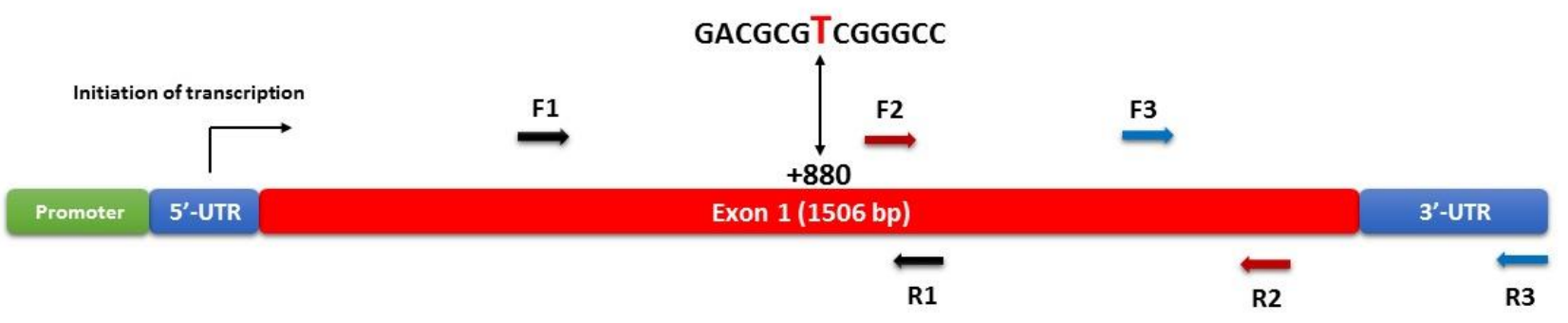

Fig. 1 A representative demonstration of the FOXC2 gene structure, primers used, amplified regions and the detected mutation. F1R1 refers to the forward-1 primer and Reverse-1 primer (576 bp). F2-R2 refers to the forward-2 primer and reverse-2 primer (515 bp). F3-R3 refers to the forward-3 primer and reverse-3 primer (463 bp). 


\section{PCR amplicon Purification and Sequencing}

The PCR products for the amplified regions were purified by the EZ-10 spin column PCR purification kit (Bio Basic, Canada) according to the manufacturer's instructions. The sequencing procedure was carried out at Princess Haya Biotechnology Center-King Abdullah University Hospital using Bigdye terminator V 3.1 Cycle Sequencing kit (Applied Biosystems, Foster City, CA, USA). Followed by purification by NucleoSEQ sequence reaction cleaning kit (Macherey Nagel Ltd, Dueren, Germany). Finally, the sequencing was performed on a 3130xl genetic analyzer (Applied Biosystems, Foster City, CA, USA). Chromatogram of the sequence products was analyzed by ChromasPro software (Version 1.42, Technelysium Pty, Ltd.)

\section{RESULTS}

The thirty-two individuals from eight families included 20 $(62.5 \%)$ males and 12 females aged between 20 to 70 years old, with an average age of 33 years. Nineteen individuals (59\%), including thirteen males and six females, exhibited symptoms of hemorrhoids. Eight individuals (25\%) manifested varicose veins symptoms including five males and three females. Five individuals (16\%) (two males and three females) had both varicose veins and hemorrhoids. Finally, four subjects $(12.5 \%)$ had a history of myocardial infarctions. Disease onset age ranged between 20 and 55 years with an average of 29 years.

The target sequences located between +444 and +1784 positions in the FOXC2 gene were amplified by specific primers and resolved by gel electrophoresis. The specific size of each amplicon was confirmed by comparing it with the appropriate ladder size as shown in fig. 1. The first amplicon is presented in figure $1 \mathrm{~A}$, showing the size of $576 \mathrm{bp}$. While the second amplicon is presented in figure $1 \mathrm{~B}$, showing the size of $515 \mathrm{bp}$. Besides, the third amplicon is presented in figure $1 \mathrm{C}$, showing the expected size of $463 \mathrm{bp}$. Analyzing the chromatograms of all samples, one insertion mutation (Fig. 2) was found in two subjects of the same family at the coding sequence located in the first amplified segment. Direct sequencing confirmed the presence of the point mutation (Fig. $3)$. The mutation was homozygous for one base pair insertion at 880 '881insT, where thymine is inserted between 880 guanine and 881 cytosine, creating a frameshift mutation. The frequency of the detected frameshift mutation represents $6 \%$ of the affected individuals. The two subjects are brother and sister and no samples could be obtained from their parents since the mother was deceased and the father was unwilling to participate in this study. Interestingly, the reported frameshift mutation was detected in individuals with hemorrhoids, varicose veins and myocardial infarction (F71) H+VV, Severe)(MI) F72 (H+VV, Sever)(MI)) (Table 2).
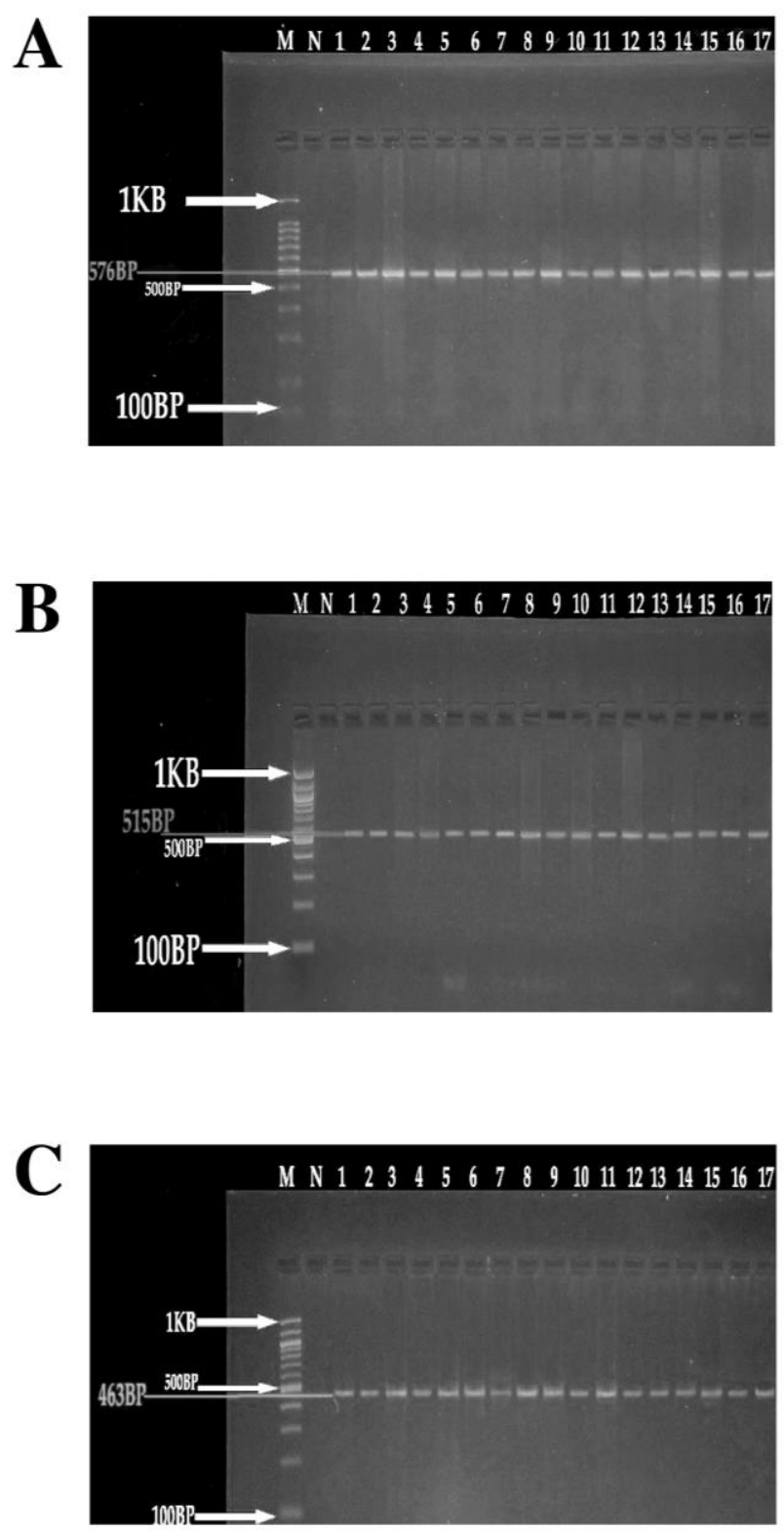

Fig. 2 A) Amplified PCR products resolved on 2\% agarose gel from varicose veins and hemorrhoids patients using $1 \mathrm{~F}$ and $1 \mathrm{R}$ primers that amplify $576 \mathrm{bp}$. Lane 1-17 show representative $\mathrm{PCR}$ products $\mathrm{B}$ ) Using $2 \mathrm{~F}$ and $2 \mathrm{R}$ primers that amplify $515 \mathrm{bp}$. C) Using $3 \mathrm{~F}$ and $4 \mathrm{R}$ primers that amplify $463 \mathrm{bp}$. Lane M: $100 \mathrm{bp}$ DNA ladder, Lane $\mathrm{N}$ is a negative control.

Table 2. Distribution and frequency of mutations in affected individuals

\begin{tabular}{|c|c|c|c|c|}
\hline Mutation & $\begin{array}{c}\text { Family } \\
\text { no. }\end{array}$ & $\begin{array}{c}\text { Type of } \\
\text { mutation }\end{array}$ & Subject I.D & $\begin{array}{c}\text { Freq } \\
\text { uency }\end{array}$ \\
\hline $\begin{array}{c}\text { 880'881in } \\
\text { sT }\end{array}$ & $\begin{array}{c}\text { Family } \\
7\end{array}$ & $\begin{array}{c}1 \text { bp } \\
\text { insertion }\end{array}$ & $\begin{array}{c}\text { F71(H+VV, } \\
\text { Severe)(MI) } \\
\text { F72(H+VV, } \\
\text { Sever)(MI) }\end{array}$ & $6 \%$ \\
\hline
\end{tabular}




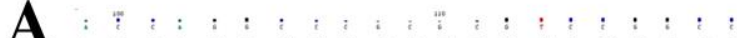
A C C A G G C C C G C G C G T C C G G C C

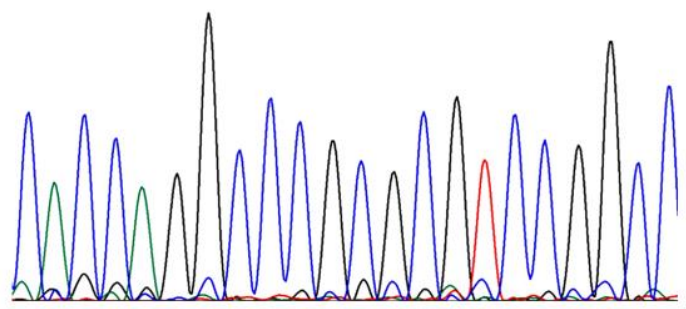

B

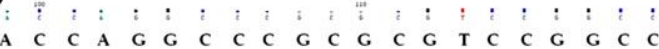

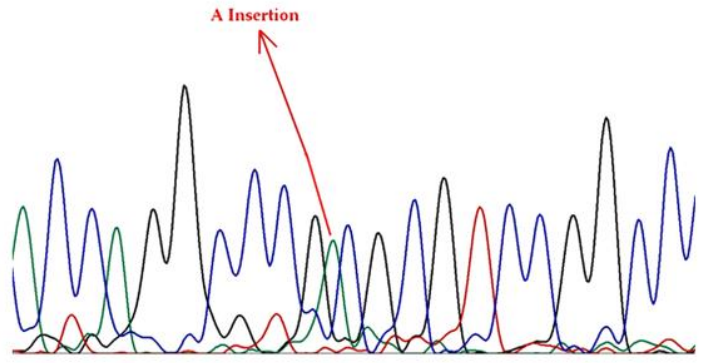

Fig. 3 Electropherogram of sample number F71 shows: (A) an adenine homozygous insertion translating into a thymine insertion at +880 bp of $F O X C 2$ gene sequence compared to $(B)$ the normal sequence.

\section{DISCUSSION}

Previous studies indicated that the FoxC2 transcription factor is a central player in the regulation and control of different developmental pathways such as skeletal, neural and vascular systems [24-27, 31-37]. Additionally, a functional study showed that embryos lacking either FoxC1 or FoxC2, exhibited abnormal phenotypes, including defects in the axial skeleton and cardiovascular system. Moreover, compound FOXC1; FOXC2 homozygote mutants die earlier with much more severe symptoms than those of a single homozygous mutant. This suggests that the genes have similar, dosedependent functions, and compensate for each other in the early development of the heart, blood vessels, and somites [42]. Moreover, vascular pathogenesis has been associated with FoxC2 expression [30]. Besides, tumor angiogenesis is regulated by the FoxC2 factor [38-40].

FoxC2 transcription factor has been proposed to be associated with the development of different vascularization complications including hemorrhoids and varicose veins [18]. Consistently, in the current study, we reported a frameshift mutation (880'881insT) in the FOXC2 gene in two siblings who manifested severe varicose veins, hemorrhoids and history of myocardial infarction. These findings support previous studies that linked the $F O X C 2$ gene alterations with different vascular pathogenesis. For instance, the D16S520 marker of the FOXC2 gene was associated with varicose veins [21]. In another study, FOXC2 mutations were reported in primary valve failure in veins of the lower limb with varicose veins [22]. Fang, et al., (2000) reported a nonsense mutation and a truncating mutation in the FOXC2 gene in the hereditary lymphedema-distichiasis [43]. As well, other studies found truncating mutations in the FOXC2 gene in patients diagnosed with multiple-lymphedema diseases [44, 45]. Also, FOXC2 mutations were found in primary venous valve failure [46] In a previous study, we found a significant association between the 5'-UTR-FOXC2 gene polymorphisms and increased prevalence of hemorrhoids and varicose veins indicating that FOXC2 gene expression regulation is associated with the development of hemorrhoids and varicose veins [18]. Currently, we reported that the FOXC2 structure is also a player in the development of blood vessel angiogenesis. This newly reported insertion mutation in the $F O X C 2$ coding region is reported in siblings displaying severe hemorrhoids and varicose veins with relatively early onset (37 and 41 at the time of the study). Both siblings needed surgical intervention to alleviate or mitigate the intensity of hemorrhoids and extended medical supervision and medication in response to the severity of varicose veins in there lower limbs. Interestingly, the affected individuals have been reported with a history of myocardial infarction (MI). Therefore, the current findings underscore the possible role of FoxC2 factor in the development of myocardial complications. Such an assumption needs more genetic studies of familial myocardial infarction. Mutation and genetic variations in different genes have been suggested to be associated with the development of cardiovascular disorders including MI [47]. However, most of these genes were related to cholesterol and lipids metabolism. For instance, certain polymorphisms in the low-density lipoprotein (LDL) receptor-related protein 8 (LRP8) and $A P O A 5$ genes were found to be associated with familial and early-onset CAD and MI [48-50].

The insertion mutation is located in the coding region of the gene, precisely at $+880 \mathrm{bp}$, leading to a frameshift beginning at $+881 \mathrm{bp}$, resulting in a premature stop codon at bp 1386. This frameshift is expected to cause a change in the peptide sequence following AA 294 and the premature stop codon was located at AA 462. The insertion may result in a significant difference in peptide sequence forming the Wing 2 (W2) portion of the protein and a possible subsequent difference in 3-dimensional structure, especially in W2. The structure of the FoxC2 protein extending from the 3rd beta-sheet, or S3, to the end of the carboxyl terminus, and is mainly responsible for high binding affinity to the DNA binding site [51]. Depending on the molecular structure of the FoxC2 transcription factor, the frameshift mutation excludes components of the FoxC2 DNA binding Helix, major helixes and connector $\beta$ strands. Therefore, the results of the current cohort suggested a molecular role of the reported frameshift mutation in the +881 site on the function of the truncated-FoxC2 transcription factor due to the significant change in the structure of W2 domain, 
consequently, leads to the development of both hemorrhoids, varicose veins and maybe MI.

Molecular studies demonstrated the role of the W2 on the FoxC2 function. For instance, how W2 influences DNAprotein is much like the arms of a brawny beast warping the DNA. W1 and W2 could provide the interactions that would bend the DNA, narrowing the major groove around the recognition helix, $\mathrm{H} 3$, in the process [52]. Alterations in this part of the binding site have a dramatic impact on DNA binding [51]. This might indicate a diminished affinity in DNA binding rather than loss of function completely considering most of the frameshift occurred at the W2 flanking sequence on the C-terminal side of the core complex.

The symptom severity in the two siblings found to have a single gene defect raises the notion that the frameshift mutation causes the FoxC2 protein to lose either its transcription-activation or binding-specificity properties. In addition, the development of $\mathrm{MI}$ in the two siblings underscores the possible role of the FoxC2 transcription factor in the pathogenesis of different vascular tissues. However, the low frequency $(6 \%)$ of the +881 frameshift mutation is limiting the outcomes of the current study. Therefore, more genetic studies are required to elucidate the role of FOXC2 genetic variations in the development of hemorrhoids, varicose veins and MI. Our findings are supported by previous findings in a study that reported another novel mutation in the FOXC2 gene at the position (c.867insA) in a family with lymphoedema of lower limbs [53].

Despite the presence of +881 frameshift mutation in the two siblings who manifested hemorrhoids, varicose veins and MI, our study is limited by the low population sample as well as the incomplete family study of the two subjects with the recorded mutation. Expanding this study to include more genes that might be of importance in normal venous development and formation including thrombomodulin and endothelin 1 is also highly recommended such as $T N F \alpha$ and insulin genes. Nevertheless, our findings shed a light on the probable role of the FOXC2 gene on the development of hemorrhoids and varicose veins. These findings suggest a possible molecular marker for the development of the aforementioned vascular disorders. Besides, the downstream genes can be associated with angiogenic disorders such as integrin $\beta 3$ and $C X C R 4$ genes [31-34]. Therefore, our findings are underscoring the importance of more genetic and molecular studies to reveal a possible genetic predisposition for the development of hemorrhoids, varicose veins and/or vascular disorders.

\section{CONCLUSION}

The results of the current study showed the presence of an 880 881InsT mutation in two subjects (siblings) with severe hemorrhoids and varicose veins symptoms constituting $6 \%$ of the study population. The insertion was located at +880 bp in the coding region and inserts thymine causing a frameshift mutation. The obvious heritability of this mutation combined with the severity of the subject's symptoms shows a possible heritable factor inducing these symptoms and the conceivable role of the FOXC2 gene in the development of hemorrhoids, varicose veins and myocardial infarction. Our findings offer a new possible genetic marker of heritable vascular disorders.

\section{ACKNOWLEDGMENT}

We are very thankful to the Deanship of Scientific Research and Graduate Studies and to Mr. Adel Rababah for his help in the fine art production

\section{REFERENCES}

1. Sun, Z. and J. Migaly, Review of hemorrhoid disease: presentation and management. Clinics in colon and rectal surgery, 2016. 29(01): p. 022-029.

2. Sandler, R.S. and A.F. Peery, Rethinking what we know about hemorrhoids. Clinical Gastroenterology and Hepatology, 2019. 17(1): p. 8-15.

3. Peery, A.F., et al., Risk factors for hemorrhoids on screening colonoscopy. PLoS One, 2015. 10(9): p. e0139100.

4. Pigot, F., L. Siproudhis, and F.-A. Allaert, Risk factors associated with hemorrhoidal symptoms in specialized consultation. Gastroenterologie clinique et biologique, 2005. 29(12): p. 1270-1274.

5. Riss, S., et al., The prevalence of hemorrhoids in adults. International journal of colorectal disease, 2012. 27(2): p. 215-220.

6. Dehn, T. and M. Kettlewell, Haemorrhoids and defaecatory habits. The Lancet, 1989. 333(8628): p. 54-55.

7. Jacobs, D.O., Hemorrhoids: what are the options in 2018? Current opinion in gastroenterology, 2018. 34(1): p. 46-49.

8. Plackett, T.P., et al., Ehlers-Danlos SyndromeHypermobility Type and Hemorrhoids. Case reports in surgery, 2014. 2014.

9. Nasseri, Y., et al., Abnormalities in collagen composition may contribute to the pathogenesis of hemorrhoids: morphometric analysis. Techniques in coloproctology, 2015. 19(2): p. 83-87.

10. Willis, S., et al., Haemorrhoids-a collagen disease? Colorectal Disease, 2010. 12(12): p. 1249-1253.

11. Serra, R., et al., Hemorrhoids and matrix metalloproteinases: a multicenter study on the predictive role of biomarkers. Surgery, 2016. 159(2): p. 487-494.

12. Segiet, O.A., et al., Biomolecular mechanisms in varicose veins development. Annals of vascular surgery, 2015. 29(2): p. 377-384.

13. Brinsuk, M., et al., Heritability of venous function in humans. Arteriosclerosis, thrombosis, and vascular biology, 2004. 24(1): p. 207-211.

14. Lee, A.J., et al., Lifestyle factors and the risk of varicose veins: Edinburgh Vein Study. Journal of clinical epidemiology, 2003. 56(2): p. 171-179.

15. CORNU- THENARD, A., et al., Importance of the familial factor in varicose disease: clinical study of 
134 families. The Journal of dermatologic surgery and oncology, 1994. 20(5): p. 318-326.

16. Cornu-Thenard, A., et al., Importance of the familial factor in varicose disease. Clinical study of 134 families. J Dermatol Surg Oncol, 1994. 20(5): p. 31826.

17. Criqui, M.H., et al., Risk factors for chronic venous disease: the San Diego Population Study. J Vasc Surg, 2007. 46(2): p. 331-7.

18. Al-Batayneh, K.M. and R.M. Al Battah, Genetic variation in the proximal 5' UTR of FOXC2 gene in varicose veins and hemorrhoids patients. Int J Integ Biol, 2008. 4(2): p. 78-80.

19. Masuno, M., et al., Ehlers-Danlos syndrome, vascular type: A novel missense mutation in the COL3Al gene. Congenital anomalies, 2012. 52(4): p. 207-210.

20. Scappaticci, S., et al., Cytogenetic studies in venous tissue from patients with varicose veins. Cancer genetics and cytogenetics, 1994. 75(1): p. 26-30.

21. $\mathrm{Ng}, \mathrm{M}$., et al., Linkage to the FOXC2 region of chromosome 16 for varicose veins in otherwise healthy, unselected sibling pairs. Journal of medical genetics, 2005. 42(3): p. 235-239.

22. Mellor, R., G. Brice, and A. Stanton, Mutations inFOXC2are strongly associated with primary valve failure in veins of the lower limb. Journal of Vascular Surgery, 2007. 46(3).

23. Miura, N., et al., MFH-1, a new member of the fork head domain family, is expressed in developing mesenchyme. FEBS letters, 1993. 326(1-3): p. 171176.

24. Iida, K., et al., Essential roles of the winged helix transcription factor $\mathrm{MFH}-1$ in aortic arch patterning and skeletogenesis. Development, 1997. 124(22): p. 4627-4638.

25. Winnier, G.E., L. Hargett, and B. Hogan, The winged helix transcription factor MFHI is required for proliferation and patterning of paraxial mesoderm in the mouse embryo. Genes \& development, 1997. 11(7): p. 926-940.

26. Furumoto, T.-a., et al., Notochord-dependent expression of MFHI and PAXI cooperates to maintain the proliferation of sclerotome cells during the vertebral column development. Developmental biology, 1999. 210(1): p. 15-29.

27. Smith, R.S., et al., Haploinsufficiency of the transcription factors FOXC1 and FOXC2 results in aberrant ocular development. Human molecular genetics, 2000. 9(7): p. 1021-1032.

28. Davis, K.E., M. Moldes, and S.R. Farmer, The forkhead transcription factor FoxC2 inhibits white adipocyte differentiation. Journal of Biological Chemistry, 2004. 279(41): p. 42453-42461.

29. Kim, S.H., et al., The forkhead transcription factor Foxc2 stimulates osteoblast differentiation. Biochemical and biophysical research communications, 2009. 386(3): p. 532-536.
30. Papanicolaou, K.N., Y. Izumiya, and K. Walsh, Forkhead transcription factors and cardiovascular biology. Circulation research, 2008. 102(1): p. 16-31.

31. Hayashi, H., et al., The Foxc2 transcription factor regulates angiogenesis via induction of integrin $\beta 3$ expression. Journal of Biological Chemistry, 2008. 283(35): p. 23791-23800.

32. Hayashi, H. and T. Kume, Forkhead transcription factors regulate expression of the chemokine receptor CXCR4 in endothelial cells and CXCL12-induced cell migration. Biochemical and biophysical research communications, 2008. 367(3): p. 584-589.

33. Avraamides, C.J., B. Garmy-Susini, and J.A. Varner, Integrins in angiogenesis and lymphangiogenesis. Nature Reviews Cancer, 2008. 8(8): p. 604.

34. Petit, I., D. Jin, and S. Rafii, The SDF-1-CXCR4 signaling pathway: a molecular hub modulating neoangiogenesis. Trends in immunology, 2007. 28(7): p. 299-307.

35. Norrmén, C., et al., FOXC2 controls formation and maturation of lymphatic collecting vessels through cooperation with NFATcl. The Journal of cell biology, 2009. 185(3): p. 439-457.

36. Dagenais, S.L., et al., Foxc2 is expressed in developing lymphatic vessels and other tissues associated with lymphedema-distichiasis syndrome. Gene Expression Patterns, 2004. 4(6): p. 611-619.

37. $\mathrm{Wu}, \mathrm{X}$. and N. Liu, FOXC2 transcription factor: a novel regulator of lymphangiogenesis. Lymphology, 2011. 44(1): p. 35-41.

38. Sano, H., et al., The Foxc2 transcription factor regulates tumor angiogenesis. Biochemical and biophysical research communications, 2010. 392(2): p. 201-206.

39. Kume, T., The role of FoxC2 transcription factor in tumor angiogenesis. Journal of oncology, 2012. 2012.

40. Mani, S.A., et al., Mesenchyme Forkhead 1 (FOXC2) plays a key role in metastasis and is associated with aggressive basal-like breast cancers. Proceedings of the National Academy of Sciences, 2007. 104(24): p. 10069-10074.

41. Kume, T., Foxc2 transcription factor: a newly described regulator of angiogenesis. Trends in cardiovascular medicine, 2008. 18(6): p. 224-228.

42. Kume, T., et al., The murine winged helix transcription factors, Foxc1 and Foxc2, are both required for cardiovascular development and somitogenesis. Genes Dev, 2001. 15(18): p. 2470-82.

43. Fang, J., et al., Mutations in FOXC2 (MFH-1), a forkhead family transcription factor, are responsible for the hereditary lymphedema-distichiasis syndrome. The American Journal of Human Genetics, 2000. 67(6): p. 1382-1388.

44. Finegold, D.N., et al., Truncating mutations in FOXC2 cause multiple lymphedema syndromes. Human molecular genetics, 2001. 10(11): p. 11851189. 
45. Erickson, R.P., et al., Clinical heterogeneity in lymphoedema-distichiasis withFOXC2 truncating mutations. Journal of medical genetics, 2001. 38(11): p. 761-766.

46. Mellor, R.H., et al., Mutations in FOXC2 are strongly associated with primary valve failure in veins of the lower limb. Circulation, 2007. 115(14): p. 1912-20.

47. Dai, X., et al., Genetics of coronary artery disease and myocardial infarction. World journal of cardiology, 2016. 8(1): p. 1.

48. Shen, G.-Q., et al., A novel molecular diagnostic marker for familial and early-onset coronary artery disease and myocardial infarction in the LRP8 gene. Circulation: Cardiovascular Genetics, 2014. 7(4): p. 514-520.

49. Shen, G.-Q., et al., An LRP8 variant is associated with familial and premature coronary artery disease and myocardial infarction. The American Journal of Human Genetics, 2007. 81(4): p. 780-791.

50. Do, R., et al., Exome sequencing identifies rare LDLR and APOA5 alleles conferring risk for myocardial infarction. Nature, 2015. 518(7537): p. 102-106.

51. Pierrou, S., et al., Cloning and characterization of seven human forkhead proteins: binding site specificity and DNA bending. EMBO J, 1994. 13(20): p. 5002-12.

52. Brennan, R.G., The winged-helix DNA-binding motif: another helix-turn-helix takeoff. Cell, 1993. 74(5): p. 773-6.

53. Rucigaj, T.P., et al., A novel mutation in the FOXC2 gene: a heterozygous insertion of adenosine (c. 867insA) in a family with lymphoedema of lower limbs without distichiasis. Radiology and oncology, 2017. 51(3): p. 363-368.
Creative Commons Attribution License 4.0 (Attribution 4.0 International, CC BY 4.0)

This article is published under the terms of the Creative Commons Attribution License 4.0

https://creativecommons.org/licenses/by/4.0/deed.en_US 